[Technical Paper]

\title{
Self-Written Waveguide Technology with Light-Curable Resin for Easy Optical Interconnection
}

\author{
Osamu Mikami*, Tadayuki Enomoto**, Yukinobu Soeda***, and Chiemi Fujikawa* \\ *School of Engineering, Tokai University, Hiratsuka, Kanagawa 259-1292, Japan \\ **School of Integrated Design Engineering, Keio University, Hiyoshi, Kohoku-ku, Yokohama, Kanagawa 223-8522, Japan \\ ***Center for Nano Materials and Technology, School of Materials Science, Japan Advanced Institute of Science and Technology, Nomi, Ishikawa \\ 923-1292, Japan
}

(Received July 25, 2014; accepted October 14, 2014)

\begin{abstract}
The success of smart optical interconnects for practical use strongly depends on the development of sophisticated coupling technologies that can achieve both high coupling efficiency and easy alignment. One promising technology for solving these problems is the fiber self-written waveguide (SWW) method, which uses light-curable resin. We fabricated a micro-scale $90^{\circ}$ light-path converter on the top of mechanically transferable connector that enables smart connection between fibers and an optical wiring board. Then, building upon this fiber SWW technology, we devised a new technology called the mask-transfer SWW method. By applying this technology, we developed a new polymer V-groove fabrication method, which enables V-grooves to be formed at designated positions to facilitate coupling between fibers and devices.
\end{abstract}

Keywords: Optical Interconnect, UV-curable Resin, Self-written Waveguide, Polymer V-groove, Mask-transfer Method

\section{Introduction}

Optical interconnects have attracted much attention as a solution to the metallic wiring bottleneck. Recently, equipment for signal processing and information transmission has become faster. As a consequence, metallic wiring is approaching the limit of its capabilities, and optical interconnects are needed in board-to-board interconnection in order to overcome this bottleneck.[1-3] However, optical wiring has some limitations. For example, high optical coupling efficiency requires extremely precise alignment. To develop practical optical interconnects, sophisticated packaging and coupling technologies are needed that can achieve both high coupling efficiency and easy alignment.

The fiber self-written waveguide (SWW) method is a promising approach to solving these problems. $[4,5]$ The fiber SWW method uses a light-curable resin and a fiber. An optical channel waveguide can be fabricated by irradiating a UV-light and/or a blue laser beam into the resin from the fiber facet. This optical waveguide is formed in the shape of an icicle from the end face of the optical fiber. Inherent to this technology is the very high coupling efficiency achieved between the fiber and the fabricated wave- guide core. Building upon the fiber SWW technology, we devised a new technology called the mask-transfer SWW method.[6-8] This method involves contact exposure of UV-curable resin through a photomask. A unique aspect of this method is that SWWs having two-dimensional array patterns can be fabricated by a single shot of a UV beam.

We used these methods to fabricate and test several new optical interconnect devices. First, we introduce a microscale $90^{\circ}$ light-path conversion device attached to a mechanically transferable (MT) connector. We fabricated this new device by the fiber SWW method.[9] Second, we propose a new V-groove fabrication method that applies the mask-transfer SWW method.[10] The use of V-grooves is a well-known technique for aligning optical fibers. The mask-transfer SWW method enables V-groove formation at designated positions to facilitate coupling between fibers and devices.

\section{Fabricating a $90^{\circ}$ Light-path Conversion Device by the Self-written Waveguide Method}

\subsection{Proposed device configuration}

Transmitting an optical signal into an optical fiber from a 


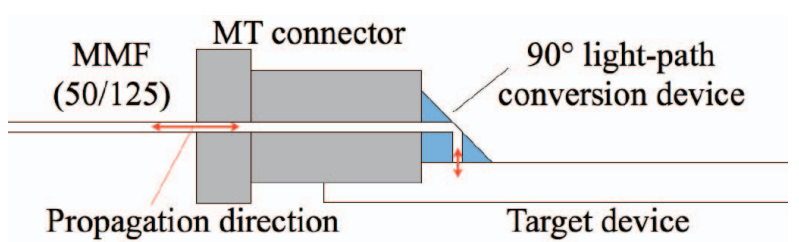

(a) Schematic diagram

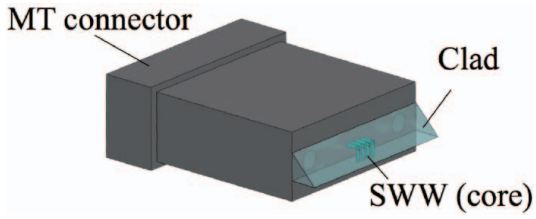

(b) Overall appearance

Fig. 1 Proposed $90^{\circ}$ light-path conversion device $(\mathrm{MMF}=$ multimode optical fiber).

vertical-cavity surface-emitting laser (VCSEL) mounted on an optoelectronic printed wiring board (PWB) requires $90^{\circ}$ light-path conversion. Figure 1 shows a schematic drawing of the proposed device, which has 4-channel SWWs.[9] These SWWs are fabricated using conventional fiber SWW technology,[4, 5] and fabricated SWWs are covered with low-index cladding resin by the mask-transfer SWW method. [6-8] Optical devices can be passively aligned by using the alignment pins of the MT connector.

\subsection{Fabrication and evalution}

We fabricated a prototype of the $90^{\circ}$ light-path conversion device. Figure 2 shows a schematic illustration of the fabrication process, the details of which are as follows.

(a) Set a $45^{\circ}$ mirror-end glass substrate and a MT connector; fill the space with light-curable core resin.

(b) Cover with a glass stopper; irradiate a blue laser beam with the optical fiber of the MT connector. Repeat four times.

(c) Wash off uncured resin after forming the 4-channel SWWs.

(d) Refill the space with light-curable resin; irradiate with UV light from a mercury-xenon lamp.

(e) Wash off uncured resin, and remove the mirror and glass stopper.

We used a $405 \mathrm{~nm}$ wavelength laser beam to cure the core resin, which has a refractive index of 1.588 at a wavelength of $850 \mathrm{~nm}$. Figure 3 shows the dimensions and appearance of the fabricated SWWs, as observed under an optical microscope. The core of the $90^{\circ}$ light-path conversion device was fabricated as designed, although the device had a slightly undulating pattern. The lengths of the fabricated core's arms are 628 and $405 \mu \mathrm{m}$. The diameter of the connector-side core is $43 \mu \mathrm{m}$, which is $7 \mu \mathrm{m}$ smaller than that of the optical fiber. Figure 4 shows photographs of a prototype SWW device after formation of the

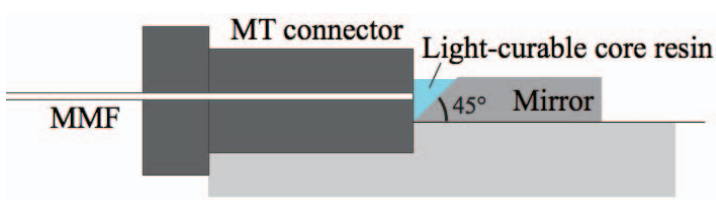

(a) Fill the space with light-curable core resin.

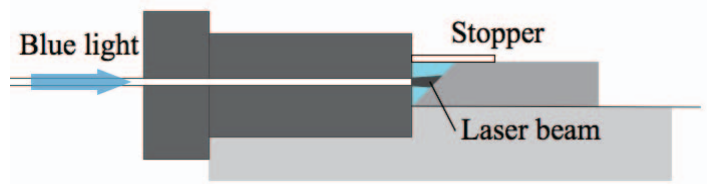

(b) Irradiate with a blue laser beam.

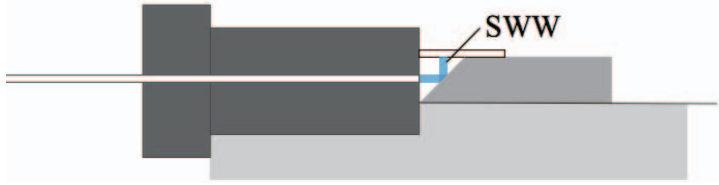

(c) Wash uncured resin.

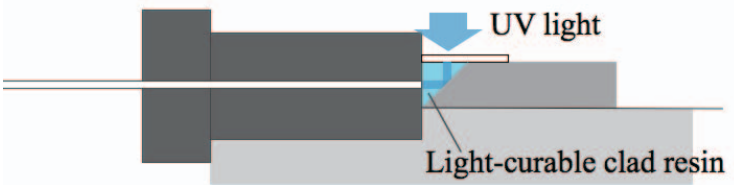

(d) Refill with light-curable clad resin and irradiate by using UV radiation

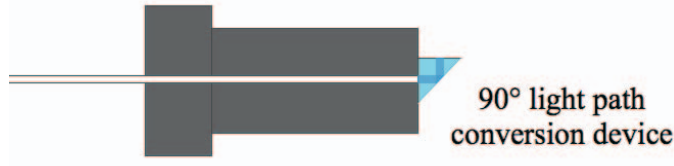

(e) Remove the mirror and glass stopper.

Fig. 2 Fabrication process.

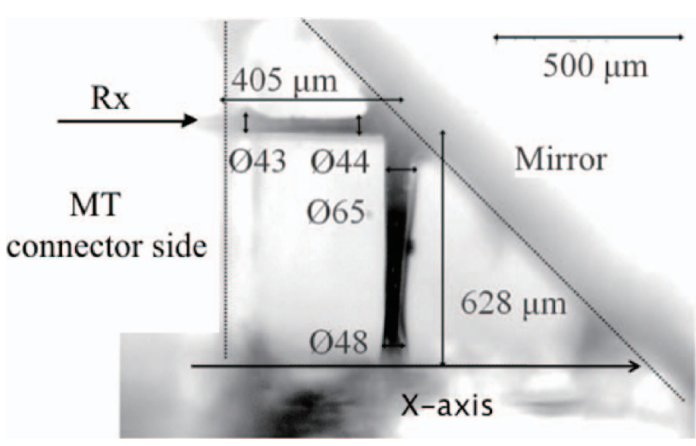

Fig. 3 Dimensions and of fabrcated SWW arms.

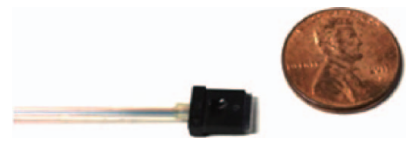

Fig. 4 Photograph of protype.

cladding.

We measured optical coupling loss with an $850 \mathrm{~nm}$ laser diode beam by setting a multimode fiber at the end face of the prototype. The losses were approximately 3.2 and 3.4 $\mathrm{dB}$ for the transmitter and receiver, respectively. The receiver is coupled into the micro prism from the MT connector side, as shown by the arrow in Fig. 3 .

\subsection{Optical loss and tolerance width}

We analyzed the optical loss and positional tolerance of 
the optical fiber for the proposed device through a raytracing simulation. The simulation results are summarized in Fig. 5. A model was constructed from SWW models with a $45^{\circ}$ mirror. We set the model dimensions to be equal to those of the fabricated device.

Optical losses of 2.28 and $1.65 \mathrm{~dB}$ were obtained at $0 \mu \mathrm{m}$ off-axis for the transmitter and receiver, respectively. In addition, the $1 \mathrm{~dB}$ down-tolerance widths of the transmitter and receiver were 15 and $25 \mu \mathrm{m}$, respectively, when the input/output fiber was moved along the $\mathrm{x}$-axis.

The simulated loss was less than the measured loss of the prototype. The optical loss of the prototype can likely be attributed to the following factors.

(1) Scattering of the irradiation beam at mirror surfaces.

(2) Non-uniform SWW shapes, for example, the undulating pattern.

Our experimental study on SWW fabrication conditions suggests that fabricating SWWs of uniform shape requires a lower-mode irradiation beam and higher optical power. Therefore, we expect that the optical coupling loss can be improved by adjusting the fabrication condition.

A major advantage of the proposed device is that a builtin alignment scheme for optical devices on the board can be incorporated into the prism. A possible scheme is shown in Fig. 6. By placing holes for the metallic pins of

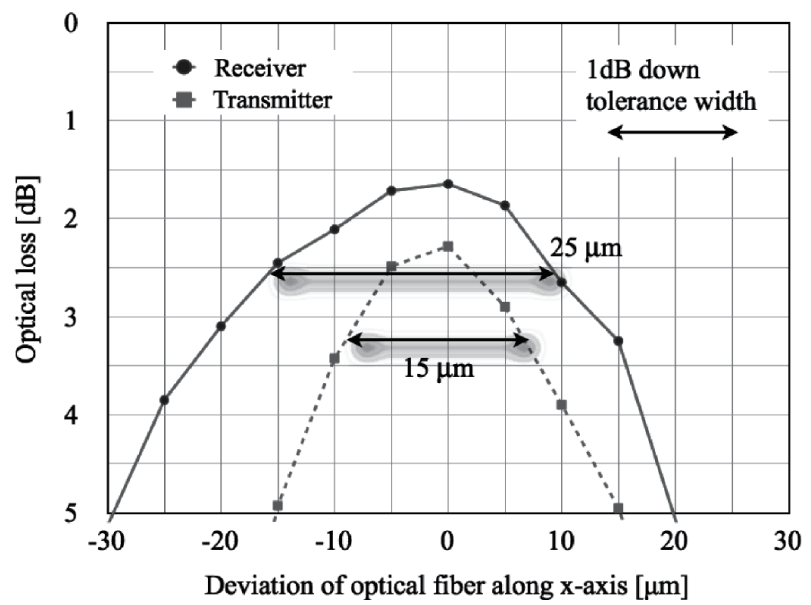

Fig. 5 Results of optical loss and positional tolerance simulation.

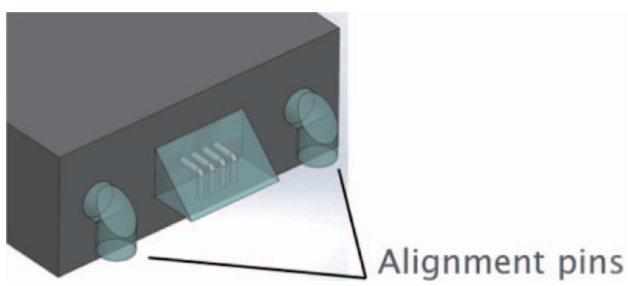

Fig. 6 Built-in alignment scheme.
MT connector, alignment pins can be formed using SWW technology, and holes for the alignment pins are prepared on the VCSEL module on the PWB. By inserting alignment pins into the holes on the VCSEL module, optical coupling can be easily accomplished. In addition, the lightpath conversion angle is not limited to $90^{\circ}$; any angle is possible by changing the mirror angle. Our study of SWW fabrication conditions suggests that SWW fabrication using a lower-mode irradiation beam and higher optical power could improve the optical coupling loss.

\section{Polymer V-grooves Prepared by the Mask-trans- fer SWW Method}

\subsection{Mask-transfer self-written waveguide method}

The mask-transfer SWW method is developed from the fiber SWW method, [6-8] and allows easy and rapid fabrication of various waveguide patterns at the same time. The basic experimental setup is shown in Fig. 7. UV-curable resin is injected between the photomask and substrate, and the thickness of the device is governed by the size of the air gap between them. The SWWs are formed at room temperature by shining UV light through the photomask. The SWW cross-section is precisely controlled by the aperture of the photomask pattern. For instance, $\mathrm{M} \times \mathrm{N}$ SWWs can be fabricated by using an $\mathrm{M} \times \mathrm{N}$ array pattern and a single shot of UV light.

\subsection{Proposed method for polymer V-grooves}

We propose a new method for preparing polymer V-grooves by the mask-transfer SWW method.[10] The fabrication process is schematically shown in Fig. 8. We first prepared a photomask that has periodic black and

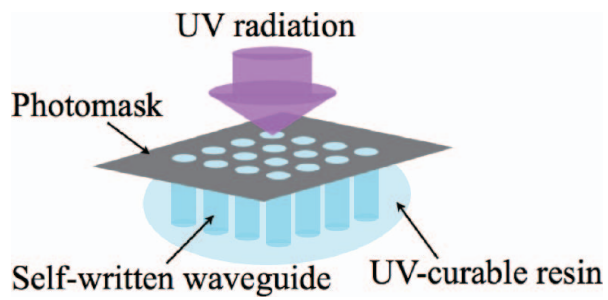

Fig. 7 Expermental setup for the basic mask-trasnfer SWW method.

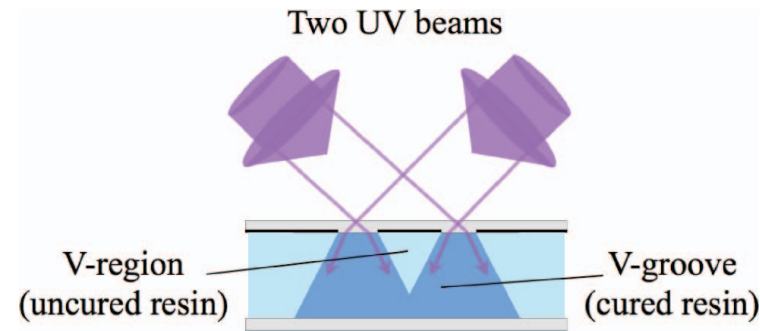

Fig. 8 Fabrication of polymer V-grooves. 
white stripes, the width of which controls the width of the V-grooves formed. When we irradiated the UV-curable resin with two UV beams at an offset angle through the mask aperture, the regions underneath the black stripes were not irradiated and remained uncured. This forms V-grooves that have a slope angle $\theta$ after removing the uncured resin.

\subsection{Mask-transfer self-written waveguide V-groove fabrication using a prism}

In the basic setup described above, the mask-transfer SWW fabrication method requires two UV beams. However, the process can be made very simple and efficient by using an isosceles prism, as shown in Fig. 9. In this way, V-grooves can be fabricated using a single UV beam. We assumed that the refractive indices of the resin, prism, and mask are the same. The slope angle $\theta$ is given by

$$
\theta=\theta_{i}-\arcsin \left\{\frac{\sin \left(\theta_{i}\right)}{n}\right\}
$$

where

$$
\theta_{i}=90-\frac{\theta_{t}}{2}
$$

Here, $\theta_{i}$ is the incident angle of the beam and $\theta_{t}$ is the top angle of the prism.

Figure 10 shows the relationship between $\theta$ and $\theta_{t}$. The shape of the polymer V-groove can be adjusted by changing $\theta_{t}$, meaning that this fabrication process is extremely flexible.

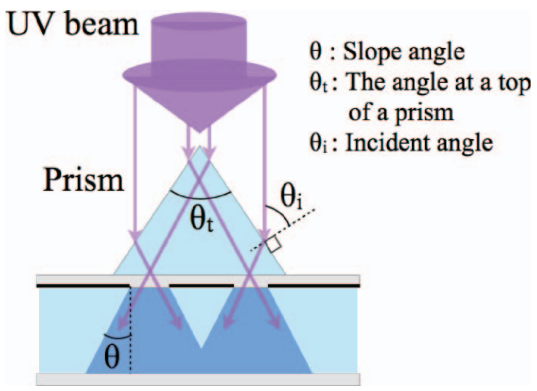

Fig. 9 Irradiation with an isosceles triangular prism.

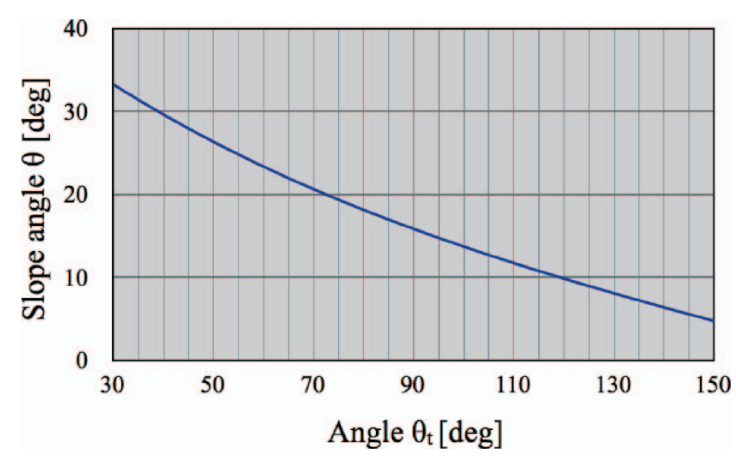

Fig. 10 Relationship between slope angle $\theta$ and angle $\theta_{t}$ at the top of the prism.

\subsection{Fabrication and evalution}

V-groove fabrication was conducted using the setup shown in Fig. 9. The height of a V-groove depends on the size of the air gap between the photomask and glass slide substrate. A UV-curable resin (DVD-003, Nippon Kayaku Co., Ltd.) was exposed to a $365 \mathrm{~nm}$ UV beam with a power density of $12.5 \mathrm{~mW} / \mathrm{cm}^{2}$ for $5 \mathrm{~s}$. In separate fabrication trials to prepare V-grooves with different shapes, two isosceles triangular prisms were used, which had $\theta_{t}$ of $60^{\circ}$ and $90^{\circ}$. Both prisms had a refractive index of 1.47 . We prepared a photomask that has alternating $50-\mu \mathrm{m}$-wide white stripes and 200- $\mu \mathrm{m}$-wide black stripes, as shown in Fig. 11. Figure 12 shows photographs of the V-grooves fabricated on glass slides by using these prisms. The results demonstrate that V-groove shape is controlled by $\theta_{t}$. Some differences were found between the theoretical values of $\theta$ and the values measured in the V-grooves. The top and bottom of the V-groove sides had maximum errors of $5-10 \mu \mathrm{m}$, and the maximum difference in the slope angle was $6.8^{\circ}$.

Figure 13 shows a photograph of an optical fiber in a V-groove fabricated with the $60^{\circ}$ prism. The position of the fiber core placed on the V-groove is shifted, which would reduce optical coupling efficiency, thus demonstrating that accurate height, width, and slope angle are required for

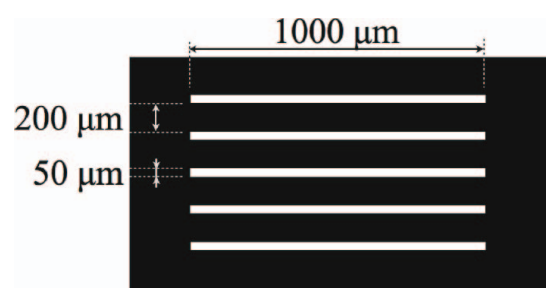

Fig. 11 Photomask with black and white stripes. (a) $60^{\circ}$ prism

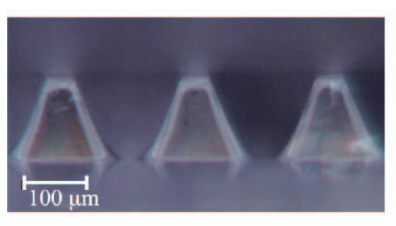

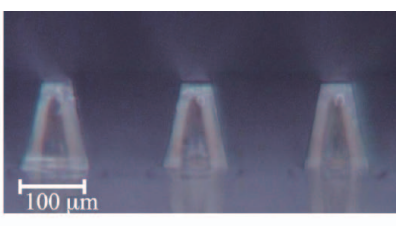

(b) $90^{\circ}$ prism
Fig. 12 Fabricated V-grooves.

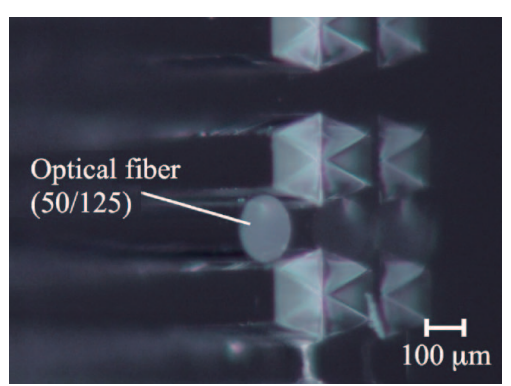

Fig. 13 Optical fiber placed on a fabricated V-groove. 
Table 1 Comparison of V-groove materials and fabrication methods.

\begin{tabular}{c|c|c|c}
\hline Substrate & Silicon wafer & Glass plate & Polymer \\
\hline Fabrication method & Wet etching & Cutting & MTSW \\
\hline Precision & High & Medium & Medium \\
\hline Cost & High & High & Low \\
\hline Utility & Practical & Practical & Research \\
\hline Productivity & Low & Low & High \\
\hline Bend & Not possible & Difficult & Possible \\
\hline Thermal stability & High & High & Medium \\
\hline $\begin{array}{c}\text { Adjustment of } \\
\text { V angle }\end{array}$ & Difficult & Possible & Easy \\
\hline Package order & V-groove first & V-groove first & Device first \\
\hline
\end{tabular}

V-groove fabrication. The fabrication errors seem large at present; nevertheless, the polymer V-grooves fabricated by this method could be used in multimode fiber systems. It is important to reduce the fabrication errors in the shape of the V-grooves for future optical systems and other applications. The cause of the fabrication errors has not yet been identified, although it is probably due to poor UV collimation and error in the angle of beam incidence on the prism. We expect that the reproducibility of V-groove shape can be substantially improved by optimizing the fabrication conditions. Our ultimate goal is that this method can be used for single-mode fiber systems.

\subsection{Advantages of mask-transfer self-written wave- guide $\mathbf{V}$-groove fabrication}

Table 1 compares fabrication methods for V-grooves. Conventional fabrication methods require sophisticated technology, thus resulting in high costs. In contrast, the mask-transfer SWW method uses a simple room-temperature process and an inexpensive resin. Of particular note is that V-grooves can be fabricated anywhere on the substrate, even after the device is assembled.

\section{Conclusion}

We have proposed and demonstrated two optical interconnect devices fabricated using fiber SWW and masktransfer SWW technologies with a light-curable resin. First, we proposed a microscale $90^{\circ}$ light-path conversion device fabricated by the fiber SWW method. Although further improvements in optical coupling efficiency are necessary, we confirmed that the device is feasible by raytracing simulation analysis. Our device enables easy optical coupling between optical fibers and VCSEL modules on PWBs. Next, we fabricated polymer V-grooves by applying the mask-transfer SWW method. We demon- strated that a prism can make this process simpler and more efficient: Two offset beams can be formed from a single UV beam. The shape of the V-groove can be controlled by adjusting the top angle of the prism. The optical interconnect devices fabricated using self-written waveguide technologies are promising and will lead to substantial advances in future optical interconnect devices.

\section{References}

[1] M. A. Meis, "Opto-electronic backplane technology for cost effective bandwidth management," Proc. ECTC2003, S25P7C, pp. 1073-1076, 2003.

[2] D. Huang, T. Sze, A. Landin, R. Lytel, and H. L. Davidson, "Optical Interconnects: Out of the box Forever?,” IEEE J. Sel. Top. Quant, Electron., Vol. 9, No. 2, pp. 614-623, March/April 2003.

[3] O. Mikami, "Development of Optical Surface Mount Technology,” The Journal of IEICE, Vol. 88, No. 6, pp. 428-434, 2005.

[4] S. J. Frisken, "Light-induced optical waveguide uptapers,” Opt. Lett., Vol. 18, No. 13, pp. 1035-1037, July 1993.

[5] T. Yamashita, M. Kagami, and H. Ito, "Waveguide Shape Control and Loss Properties of Light-Induced Self-Witten (LISW) Optical Waveguides," J. Lightwave Tech., Vol. 20, No. 8, pp. 1556-1562, August 2002.

[6] Y. Obata, M. Kanda, and O. Mikami, "Self-Written waveguide on a VCSEL-emitting window using a photomask transfer method," IEEE Photon. Tech. Lett., Vol. 18, No. 12, pp. 1308-1310, 2006.

[7] Y. Obata, Y. Oyama, H. Ozawa, T. Ito, O. Mikami, and T. Uchida, "Multi-array Self-written Waveguides using Photomask for Optical Surface Mount Tech- 
nology,” ICEP2005, pp. 225-229, April 2005.

[8] O. Mikami, Y. Mimura, H. Hanajima, and M. Kanda, "Optical Connection with Optical Pins and Self-Written Waveguides for Board-level Optical Wiring," IEICE Trans. on Electron., Vol. E90-C, No. 5, pp. 1071-1080, May 2007.

[9] Y. Soeda, T. Enomoto, and O. Mikami, "Self-Written Waveguide Technology with Light-curable Resin
Enabling Easy Optical Interconnection,” IEEE CPMT Symp. Japan 2013, Kyoto Univ. Nov, 11-13, 2013.

[10] T. Enomoto, Y. Soeda, and O. Mikami, "Optical connecting devices fabricated by self-written waveguide technology for smart optical interconnect," SPIE Photonics West, Optical Interconnects XIV, 8991-39, Paper No. OE112-26, Feb. 4-9, 2014.

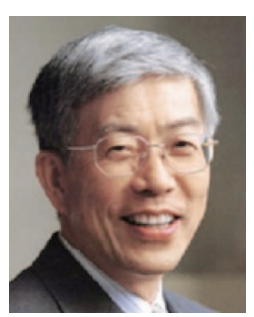

Osamu Mikami received his B.S., M.S., and Ph.D. in applied physics from Osaka University in 1969, 1971, and 1978, respectively. In 1971, he joined the Nippon Telegraph and Telephone Public Corporation (now NTT), where he was engaged in research on reversible holographic storage, optical waveguide modulators using $\mathrm{LiNbO}_{3}$ single crystals, and optical functional devices based on III-V semiconductors for 23 years. From 1985 to 1986, he spent a year at the Department of Electronic and Electrical Engineering, Glasgow University, Scotland, UK, as a Scientific Engineering Research Council visiting research fellow. From 1991 to 1992, he was a visiting associate professor at the Research Center for Advanced Science and Technology, University of Tokyo. In 1994, he left NTT and joined Tokai University as a professor in the School of Engineering, where he started new research on optical interconnection and optical wiring in printed wiring boards. In 2006, he was a visiting professor at the University of Kassel, Germany, for 4 months. In 2012, he retired from Tokai University. Now he is active in research on optical interconnection as a contract professor at Tokai University.

He first proposed the new concept of an "optical pin" for connecting between an optical fiber and optical wiring, and later proposed a new technology called mask-transfer self-written waveguides, which enables mass production of polymer optical pins in two-dimensional array structures.

He is a member of Japan Institute of Electronics Packaging and the Japan Society of Applied Physics (JSAP), as well as a Fellow of the Institute of Electronics, Information, and Communication Engineers of Japan (IEICE).

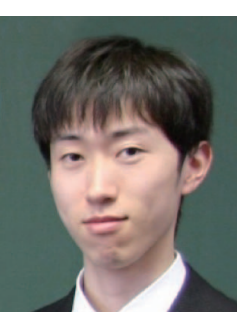

Tadayuki Enomoto received his B.E. from Tokai University in 2014. Now he is a graduate student at the School of Integrated Design Engineering, Keio University.

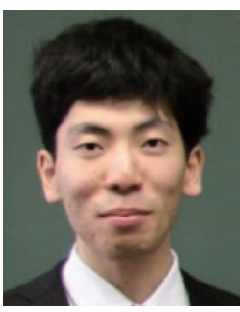

Yukinoibu Soeda received his B.E. from Tokai University in 2014. Now he is a graduate student at the Center for Nano Materials and Technology, School of Materials Science, Japan Advanced Institute of Science and Technology, Ishikawa, Japan.

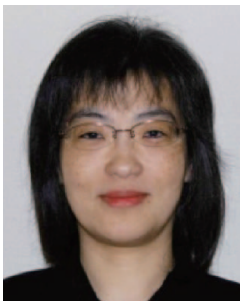

Chiemi Fujikawa received her B.E. from Japan Women's University, Tokyo, Japan, in 1992, her M.E. from the Japan Advanced Institute of Science and Technology, Ishikawa, Japan, in 1995, and her Ph.D. from Toyota Technological Institute, Nagoya, Japan, in 1999.

From 2000 to 2001, she worked as a researcher at the Telecommunications Advancement Organization of Japan in Tokyo. She was a research associate at Tokyo Polytechnic University, Kanagawa, Japan, from 2001 to 2005. She was a research associate at Japan Women's University from 2005 to 2006. From 2006 to 2011, she was a junior associate professor in the School of Engineering, Tokai University, Kanagawa, Japan. Since 2011, she has been an associate professor in the School of Engineering, Tokai University. Her main research interests include optical functional devices, optical fibers, wireless communications, and optical interference methods.

She is a member of the JSAP, the Optical Society of Japan, and the IEICE. 